\title{
Energy-Saving Technology in Indoor LTE System with DTX Based on CQI
}

\author{
Zhenyu Yang, Yong Shang, Dingyang Chen, Xiguang Zhang \\ Communication System and Signal Processing, Peking University, Beijing, China \\ Email: yangzhenyu 378@163.com, shangyong@pku.edu.cn
}

Received March 2015

\begin{abstract}
In LTE (Long Term Evolution) system, CQI (Channel Quality Indication) is a technical method to realize adaptive modulation and coding. The dispatcher of BS (base station) would allocate resource blocks to users dynamically based on CQI of uplink and downlink channels, which is necessary in the process of uplink and downlink transmission [1]. When the CQI level of the channel is high, the energy efficiency is high. On the contrary, when the CQI level of the channel is low, the energy efficiency is low. However, if signals are not transmitted until the quality of the channel is good, energy loss may be lowered in the expense of group delay. That is to say, we take CQI into account on the basis of the technology of DTX [2] [3] (Discontinuous Transmission). According to the result of simulation, the energy consumption can be reduced by using our proposed scheme. Particularly, the energy-saving effect is significant when the load is low.
\end{abstract}

\section{Keywords}

LTE System, CQI, Adaptive Modulation and Coding, Indoor Channel, Energy-Saving

\section{Introduction}

With the constant expansion of the communication networks and the improvement of communication technology, the energy consumption of communication networks is increasing rapidly [4]. Thus, green communication has become an important area of research. When analyzing the energy consumption of wireless access networks, early research has found out that the energy usage of the BS takes more than half of the total energy consumption [5]. Thus, reducing the energy usage of the BS is very important in green communication. This article will introduce a kind of technology that can reduce the energy consumption of BS.

\section{Methodology}

\subsection{CQI of LTE System}

In modern communication system, BS can select appropriate modulation and coding method to transmit data based on the CQI fed back by mobile terminals. According to 3GPP long-term evolution technology standard,

How to cite this paper: Yang, Z.Y., Shang, Y., Chen, D.Y. and Zhang, X.G. (2015) Energy-Saving Technology in Indoor LTE System with DTX Based on CQI. Int. J. Communications, Network and System Sciences, 8, 50-57. 
mobile terminals would find out the optimal modulation and coding method with BLER of less than $10 \%$ in the first transmission of HARQ, based on the value of SINR of PRB measurement group. The mobile terminals would also provide feedbacks on the indications of channel quality based on the CQI level [6]. Figure $\mathbf{1}$ and Figure 2 show the relationship between the throughput and SNR of the system, and the relationship between the BLER (Block Error Ratio) and SNR of the system.

According to Figure 1, as the SNR increases, the throughput increases with the CQI level improves. According to the Figure 2, as the SNR increases, the BLER decreases with the CQI level improves.

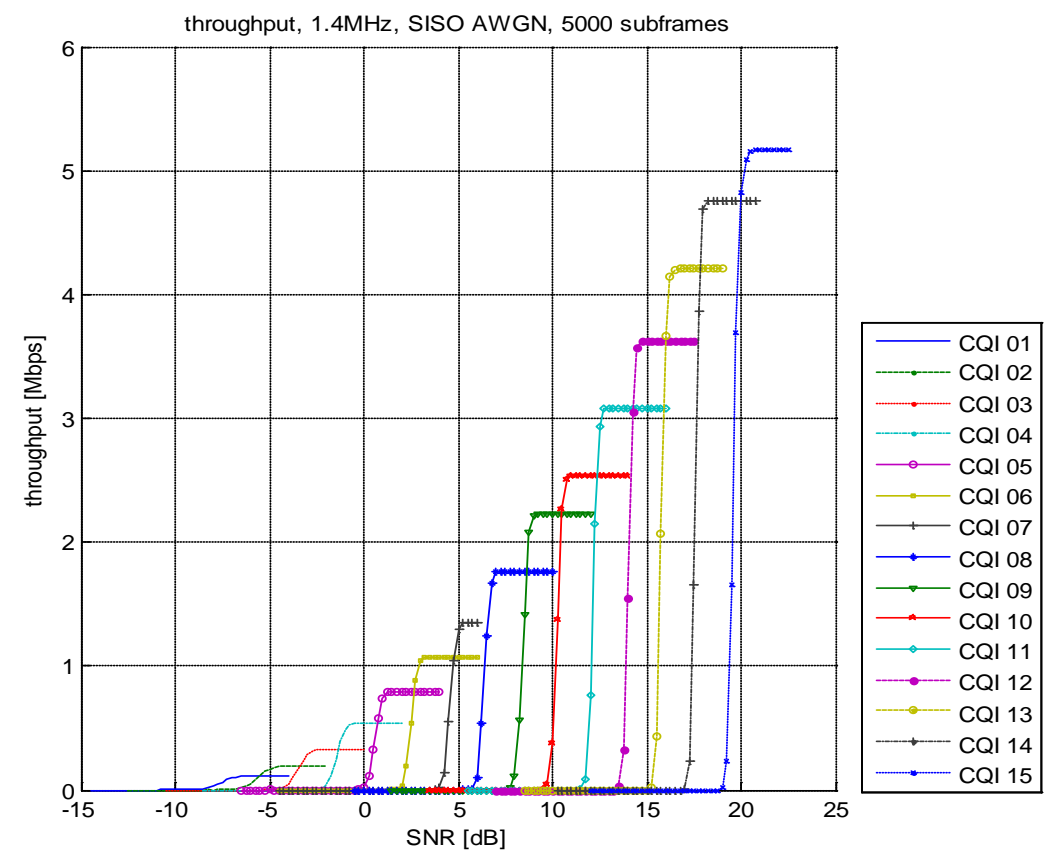

Figure 1. Throughput versus SNR under different CQI.

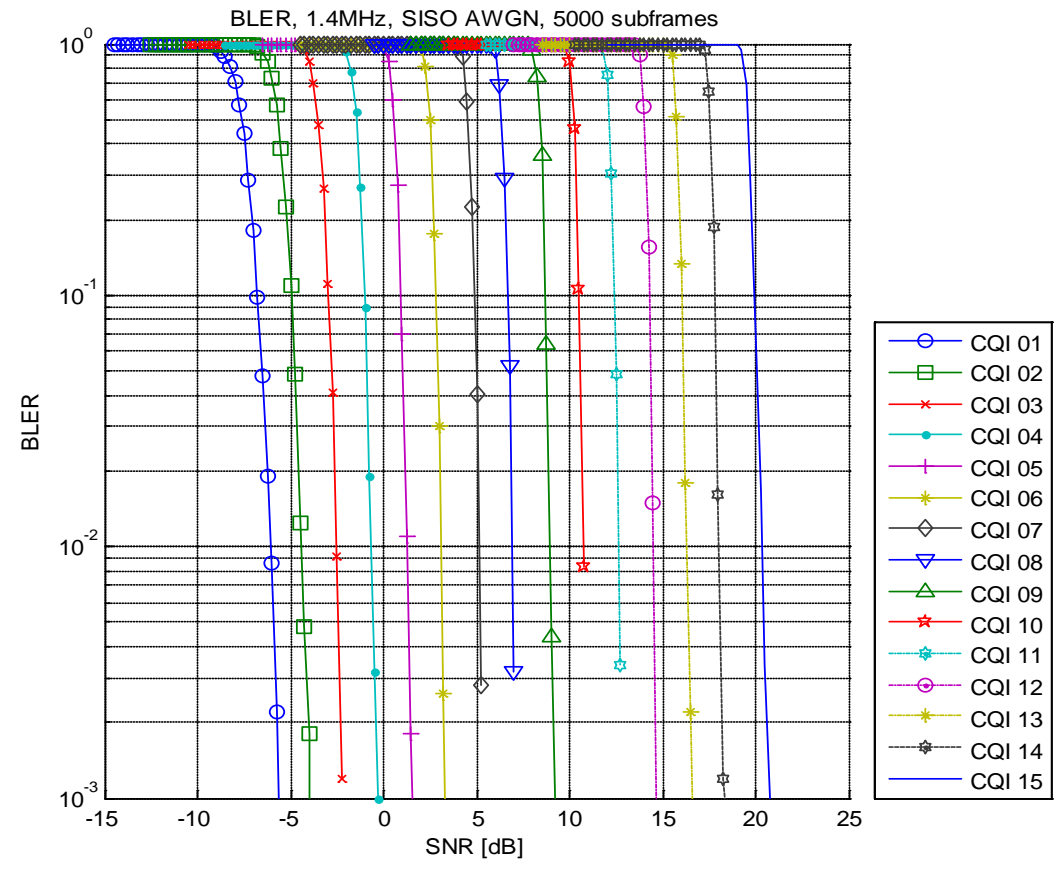

Figure 2. BLER versus SNR under different CQI. 
When the value of CQI is 1 , coding efficiency is only 0.1523 . When the value of CQI is 15 , coding efficiency is 5.5547, which is 50 times larger than the former. Towards different sub-carriers and different time points, the value of CQI changes with the condition of the channel. The total CQI is synthesized from CQI of all time periods. In order to adapt the performance limits of BER (BLER $<10 \%$ ), the system would select appropriate modulation and coding method. When the quality of the channel is relatively good, CQI index is higher and the energy efficiency of the system is higher. On the contrary, when the quality of the channel is worse, CQI index is lower, which results in energy waste.

However when the condition of the channel is poor, the transmission of data packets stops until the condition of the channel improves. The process of the transmission may also proceed in low speed after a long time of waiting to guarantee timeliness and integrity of communication system.

Figure 3 [7] demonstrates the CQI level of three users in a period of time. According to Figure 3, the value of CQI varies with time regularly but the value is not constant. Therefore, the system can choose to provide service only in a specific time period with the optimal channel conditions. In this way, energy consumption of the system or network can be reduced. Obviously, the main parameter in this technology is the CQI indication threshold of transmission suspension. If the value of CQI is lower than the threshold, which indicates poor condition of communication system, the base station would stop transmitting data to receivers.

Figure 4 [8] demonstrates the instantaneous and average values of energy consumption under different CQI. Reduction of energy consumption is 1\%, 5\%,14\% and 33\% respectively for the CQI level of 9, 11, 12 and 13.

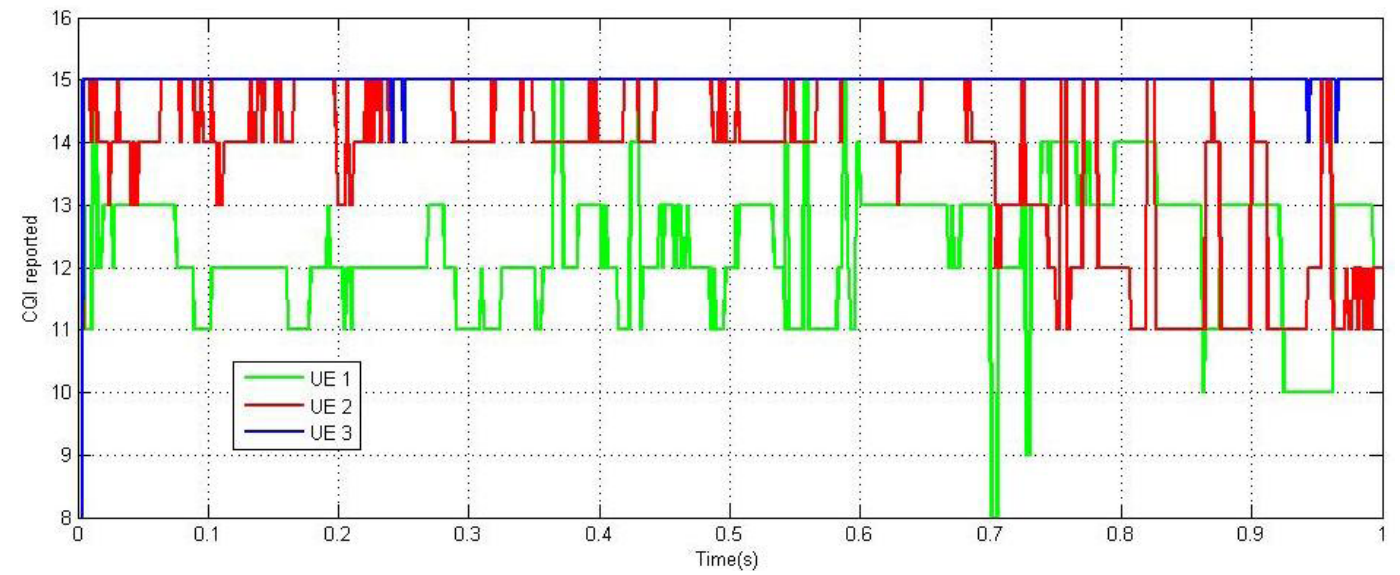

Figure 3. CQI level of different users.

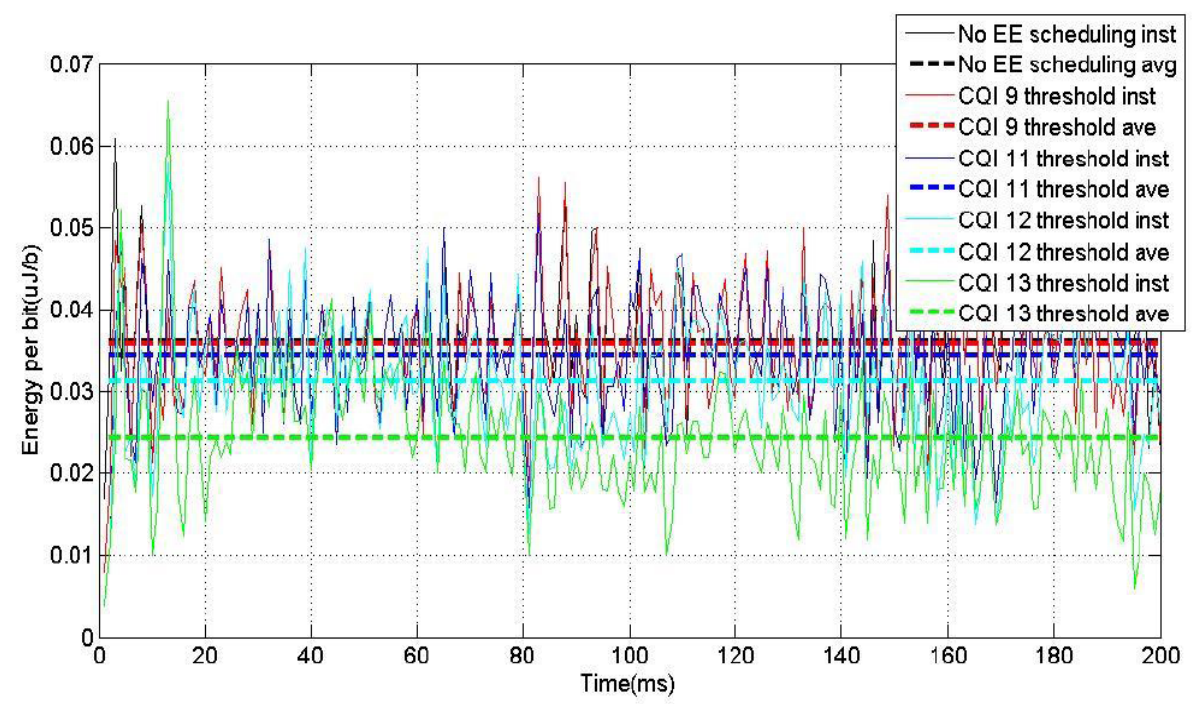

Figure 4. Energy consumption per bit under different CQI. 
The condition of indoor channel is different from the condition of outdoor channel. The power of indoor transmission is lower with less environmental variation and its coverage distance is shorter. The fading rate of indoor channel is slow and flat, therefore its CQI indication variation is less frequent compared with outdoor channel. Apparently, the condition of indoor channel are relatively better, therefore ignoring several sub-frames with low CQI level when the condition of the channel is good does not pose much influence on the quality of communication. By doing this, the energy consumption of base stations can be reduced. DTX in a short time is applicable when the load of network is low, under which situation the system is allowed to stay in dormant mode with low time delay until the quality of the channel turns good. Therefore, the maximum delay is often set to millisecond.

\subsection{Distribution of Energy Consumption of BS}

Figure 5 [9] demonstrates the energy consumption of base station. In indoor LTE system, approximately 39\% energy is consumed by baseband processor and 32\% is consumed by PA (Power Amplifier). Therefore, in indoor communication environment, baseband processing is stopped and the work of power amplifier is suspended when the load of cell reduces constantly for a long time, which is beneficial for energy saving of the system. Without reducing the QoS (Quality of Service) of indoor users, some useless wireless resource (for instance, power amplifier) in a frame can be shut down. Cell can dynamically select some sub-frames according to the need of load to transmit users' data and control information. In the rest sub-frames, dormant mode should be performed with PA shut down, which reduces the energy consumption of PA.

\subsection{DTX Based on CQI in Indoor LTE System}

For convenience of description, we assume that there's one antenna and one user. Different from outdoor channel environment, the system path loss of femtocell base station can be represented as [10]:

$$
L(\mathrm{~dB})=16.9 \log _{10}(d)+32.8+20 \log _{10}\left(f_{c}\right)
$$

$d$ represents the radius of cell, $f_{c}$ represents the frequency of carrier. The corresponding gain of the channel can be represented as:

$$
g_{p l}=10^{\frac{-L}{10}}=10 \frac{-16.9 \log _{10}(d)-32.8-20 \log _{10}\left(f_{c}\right)}{10}
$$

According to different required operation temperature $T$, the density of noise can be represented as:

$$
N_{0}(\mathrm{~W} / \mathrm{Hz})=K T=1.38 \times 10^{-23} \times 290=4.0 \times 10^{-21}
$$

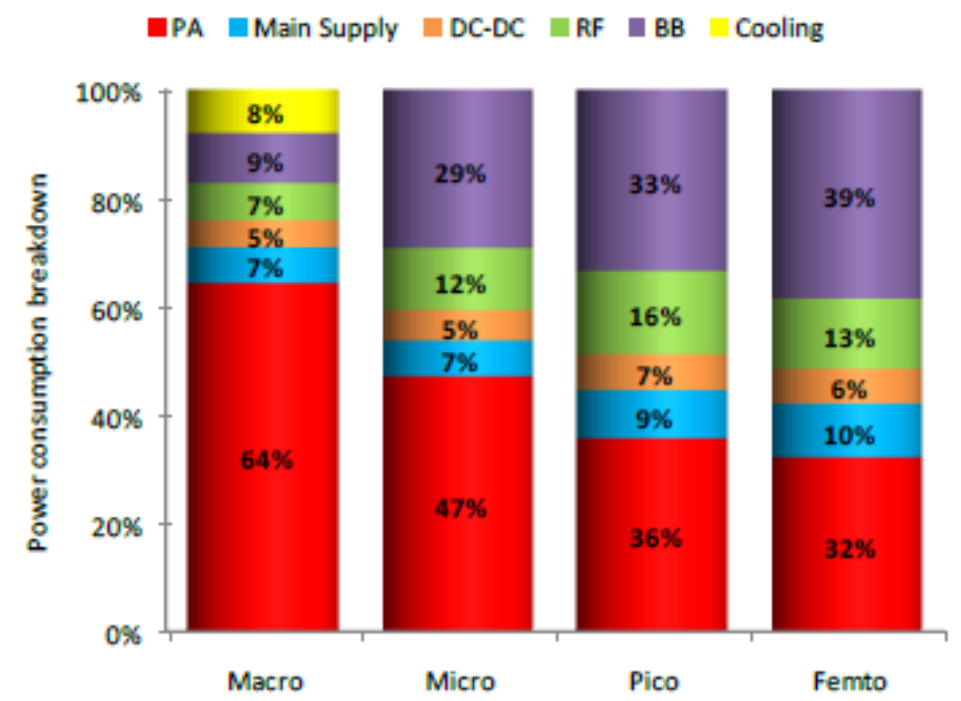

Figure 5. Distribution of energy consumption of BS. 
In the meantime, we assume that the slow-fading of channel changes in each frame. Assuming that the gain of the channel resulting from fading is $g_{f}$, the total gain of the channel can be represented as:

$$
g=g_{p l} g_{f}
$$

We simply set the radio of wireless sub-frame resource occupied by system as $20 \%$ ( $x=20 \%)$ and use the maximum power $P_{m}$ to transmit data to ensure that the information can be decoded reliably. In the meantime, we assume that fixed quantity of load is transmitted in each frame. When there's no DTX, all sub-frames in one frame have same gain of the channel and same quantity of data. According to Shannon formula, the rate of data transmission can be represented as [11]:

$$
R_{d}(\mathrm{bps} / \mathrm{Hz})=t_{d} \log 2\left(1+\frac{P_{d} g}{N_{0} B}\right)
$$

$t_{d}$ represents the time of transmission in a frame, $P_{d}$ represents the power of transmission. Therefore, $P_{d}$ can be represented as:

$$
P_{d}(\mathrm{~W})=\frac{\left(2 \frac{R_{d}}{t_{d}}-1\right) N_{0} B}{g}
$$

$t_{d}(\mathrm{~s})=(1-x) t_{f}$ and $t_{f}$ represented the length of a frame, which is $10 \mathrm{~ms}$. Therefore, the time of transmitting control signal is $t_{s}=x t_{f}$ and the energy consumption in each frame can be represented as:

$$
E(\mathrm{~J})=P_{m} t_{s}+P_{d} t_{d}
$$

When the CQI of the system is lower than the threshold, we can choose a certain number of sub-frames in each frame to transmit data while other sub-frames stop transmitting. Even if there's no data in a certain frame, we still transmit a sub-frame of control signal to contact with the mobile terminal. The value range of the number of sub-frames $N$ is 1 to 10 . Therefore, the required power of data transmission in the activated sub-frames is:

$$
P_{d s}(\mathrm{~W})=\frac{\left(2 \frac{R_{d}}{t_{d s}}-1\right) N_{0} B}{g}
$$

$t_{d s}=(1-x) t_{f} N / 10$ and $t_{s s}=x t_{f} N / 10, N$ is the number of sub-frames whose CQI level are lower than the threshold. The energy consumption of each frame can be represented as:

$$
E_{s}(\mathrm{~J})=P_{m} t_{s s}+P_{d s} t_{d s}
$$

In order to minimize the energy consumption, for the Equation (9), we do the first and second order derivative of $N$ and we can get:

$$
\begin{gathered}
\frac{\partial E_{s}}{\partial N}=\frac{P_{m} x t_{f}}{10}+\frac{\left(2^{\frac{R_{d}}{N(1-x) t_{f} / 10}}-1\right) t_{f}(1-x)}{10 g / N_{0} B}-\frac{2^{\frac{R_{d}}{N(1-x) t_{f} / 10}} \ln 2 R_{d}}{N g / N_{0} B} \\
\frac{\partial^{2} E_{s}}{\partial N^{2}}=2^{\frac{R_{d}}{N(1-x) t_{f} / 10}}(\ln 2)^{2} \frac{10 N_{0} B R_{d}^{2}}{(1-x) t_{f} g N^{3}} \geq 0
\end{gathered}
$$

Through the derivation, we know $E_{s}$ is a convex function for $N$. So for the energy consumption $E_{s}$, there is an only and best $N_{\text {opt }}$ that makes the energy consumption to the minimum. Through making $\partial E_{s} / \partial N=0$, we can get the expression of $N_{\text {opt }}$ :

$$
N_{\text {opt }}=\frac{10 \ln 2 R_{d}}{(1-x) t_{f}\left[1+\operatorname{lambertw}\left(\frac{N_{0} B x-N_{0} B+P_{m} x g}{N_{0} B(1-x) e}\right)\right]}
$$


lambertw is a lambert $\mathrm{W}$ function, namely, $f(\mathrm{~W})=W e^{W} \cdot N_{\text {opt }}$ should be an integer and the value of $N_{\text {opt }}$ may be greater than 10 when the condition of the channel is poor. In this case, the value of $N_{\text {opt }}$ should be equal to 10 .

\section{Results}

In order to validate the application effect of the energy saving technology we proposed, we define the most capacity of the transmission of the data in the system as:

$$
R_{m}(\mathrm{bps} / \mathrm{Hz})=t_{f} \log _{2}\left(1+\frac{P_{m} g}{N_{0} B}\right)
$$

So the data capacity of each sub-frame is $R_{m} / 10$, it can't reach the circumstance of $R_{d} \geq R_{m}$. We set conditions that single user, the radius of cell is $d=15 \mathrm{~m}$ and the data rate is $R_{d}=5.8 \mathrm{bps} / \mathrm{Hz}$ when the load is full ( $\mathrm{LF}=100 \%$ ) to simulate. The Figure 6 shows the energy consumption versus the number of activated subframes under different load.

As shown in Figure 6, different load corresponds to different minimum number of activated sub-frames. The bigger the load is, the bigger the number of activated sub-frames is. When the load is full ( $\mathrm{LF}=100 \%$ ), the system must activate all sub-frames to meet the requirement. When the load is zero, the energy consumption grows linearly which is consistent with our prospection.

Figure 7 shows the energy consumption versus the load under the circumstances of "DTX" and "NO DTX". From the Figure 7 we can see the energy-saving effect is significant when the load is low and the energy-saving effect becomes not obvious when the load increases. Particularly, the energy-saving effect is little when the load exceeds $60 \%$.

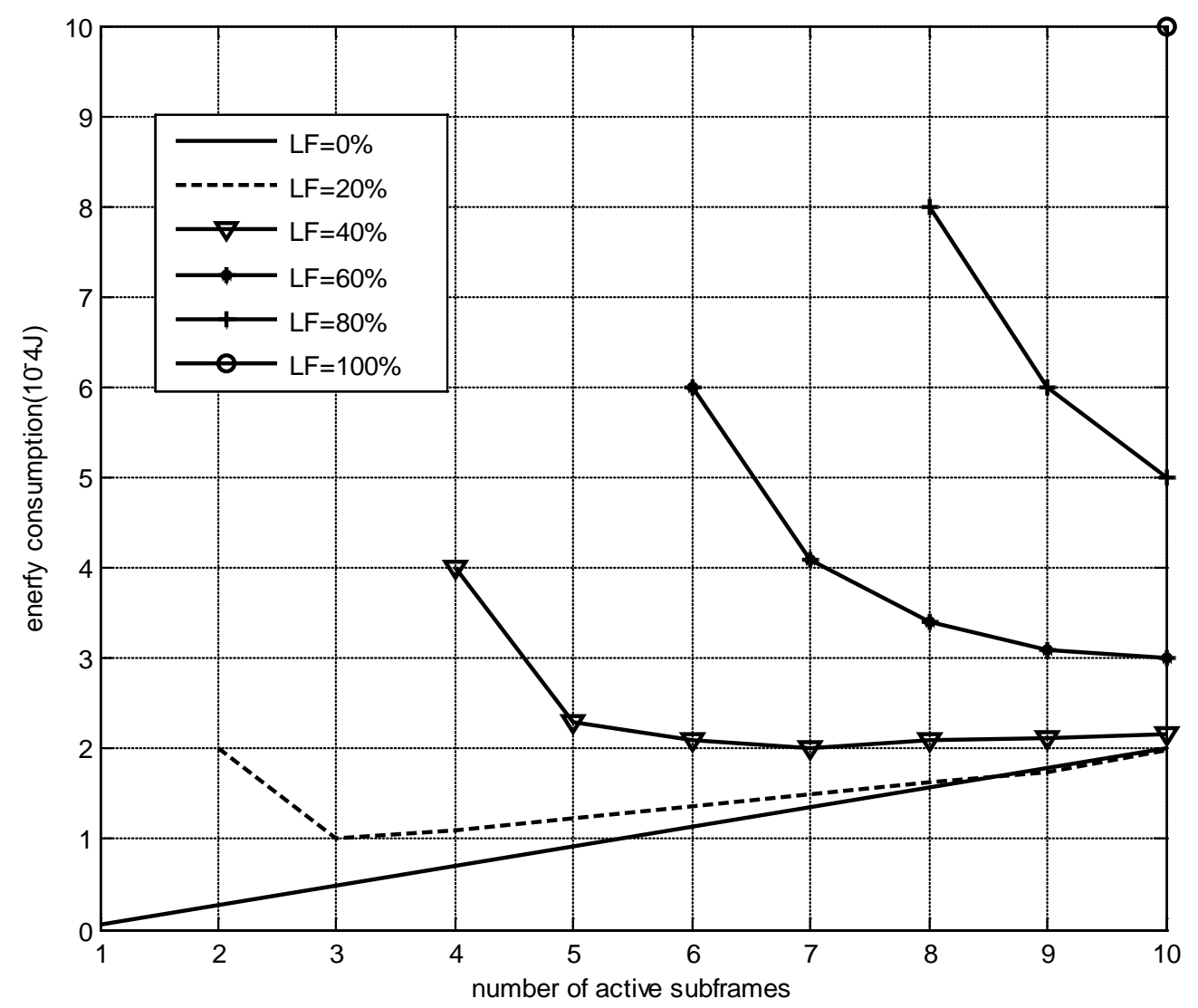

Figure 6. Energy consumption versus the number of activated sub-frames under different load. 


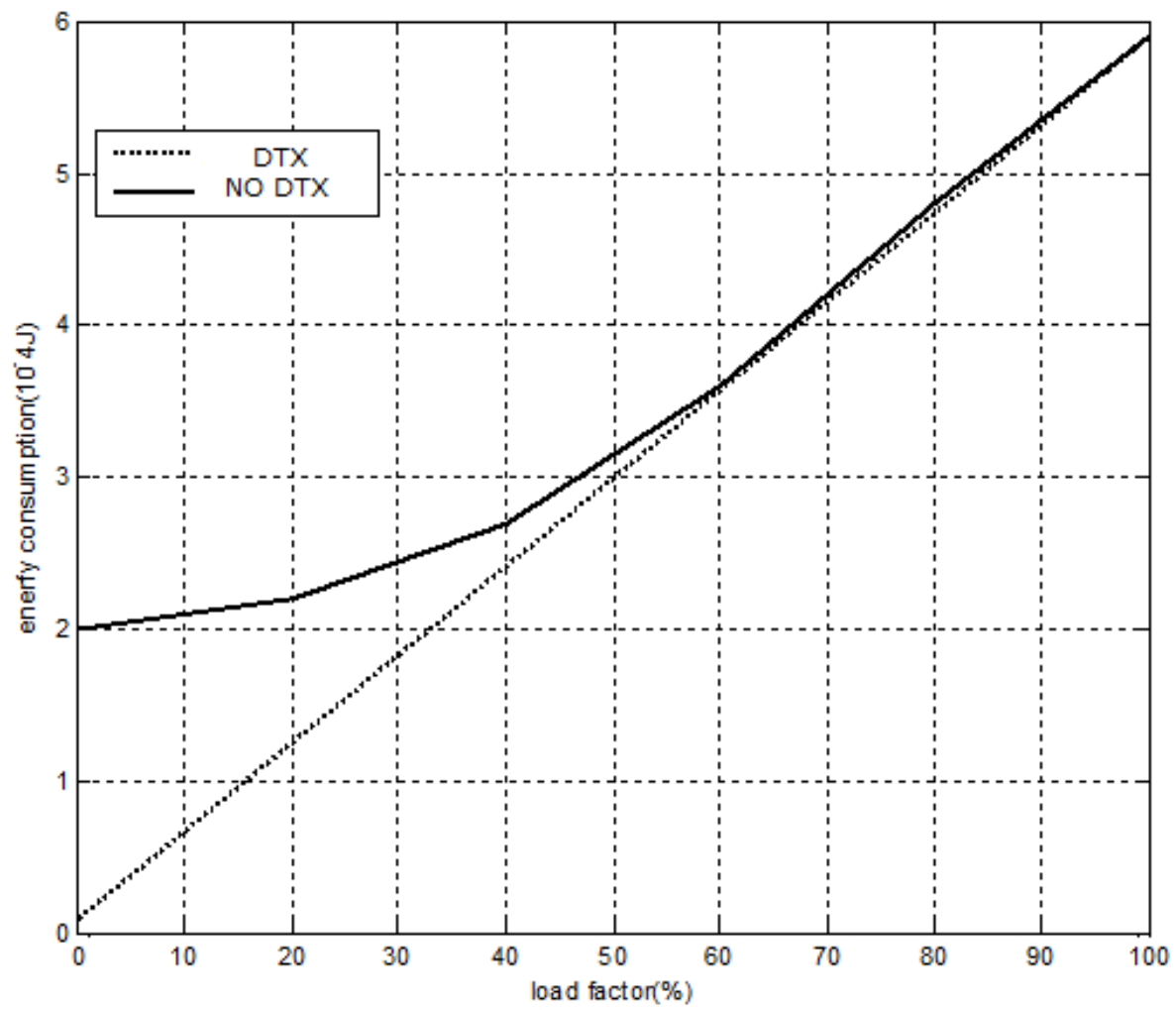

Figure 7. Energy consumption versus the load under the circumstance of "DTX" and "NO DTX”.

\section{Conclusion}

In LTE system, when the condition of the channel is poor, the energy efficiency is low. If the system stops signal transmission in a sub-frame when the CQI level is low and waits for higher CQI level to transmit, we can reduce the energy consumption at the cost of group delay. The condition of indoor channel is usually good, thus it won't bring large delay when we shut off some sub-frames, but it can reduce the energy consumption of BS. We give an algorithm to calculate the most energy-saving number of activated sub-frames. Furthermore, through simulation we show that the energy consumption can be reduced by using our proposed scheme. Particularly, the energy-saving effect is significant when the load is low. In a word, the energy-saving effect of the technology with DTX based on CQI is remarkable in indoor LTE system.

\section{Acknowledgements}

Thanks to Peking University which provides us with good environment. Particularly, the research leading to these results has received funding by the National High Technology Research and Development Program of China (SS2012AA011701). Besides, thanks to all the teachers and students who undertake the project with us because they provide us with great support.

\section{References}

[1] Zhang, X. (2008) Research of Key Technology and the System-Level Simulation of 3GPP LTE. Wuhan University of Technology, Wuhan.

[2] Shen, L.Y. and Lou, P.D. (2012) Energy-Saving Mechanism of LTE System Based on the Technology of Cell DTX. Telecom Engineering Technics and Standardization, 9, 67-72.

[3] Ericsson, ST-Ericsson (2009) Extended Cell DTX for Enhanced Energy-Efficient Network Operation. 3GPP TSGRAN WG1\#59, R1-095011.

[4] Liu, L., Pi, L.H. and Li, W.J. (2012) Development History, Development Strategy and Analysis of Policy Guidance in 
Green Communication. Mobile Communications, 9, 7-11.

[5] Chen, A.P. (2012) Current Situation of Development of Energy-Saving and the Solution in Communications Industry. Modern Science \& Technology of Telecommunications, 2, 51-54.

[6] 3GPP TS 36.213 (2009) Technical Specification Group Radio Access Network: Physical Layer Procedures (Release 8).

[7] EARTH_WP4_D4.3 (2012) Final Report on Green Radio Technologies.

[8] EARTH_WP4_D4.2 2012) Green Radio Technologies.

[9] Ziaul, H., Hamidreza, B. and Vijay, K. (2011) Green Cellular Networks: A Survey, Some Research Issues and Challenges. IEEE Communications Surveys \& Tutorials, 13, 524-540. http://dx.doi.org/10.1109/SURV.2011.092311.00031

[10] 3GPP TS 36.211 (2011) Evolved Universal Terrestrial Radio Access (E-UTRA). Physical Channels and Modulation.

[11] Samsung (2006) R1-060076: Adaptive Modulation and Channel Coding Rate. 3GPP TSG RAN WG1 LTE ad Hoc, Helsinki. www.3gpp.org 